\title{
Diagnosis of Roller Bearings Compound Fault Using Underdetermined Blind Source Separation Algorithm Based on Null-Space Pursuit
}

\author{
Lingli Cui, ${ }^{1}$ Chunguang Wu, ${ }^{1}$ Chunqing Ma, ${ }^{1}$ and Huaqing Wang ${ }^{2}$ \\ ${ }^{1}$ Key Laboratory of Advanced Manufacturing Technology, Beijing University of Technology, Chaoyang District, Beijing 100124, China \\ ${ }^{2}$ School of Mechanical \& Electrical Engineering, Beijing University of Chemical Technology, Chaoyang District, \\ No. 15 Beisanhuan East Road, Beijing 100029, China
}

Correspondence should be addressed to Lingli Cui; acuilingli@163.com and Huaqing Wang; wanghq_buct@hotmail.com

Received 10 September 2014; Revised 9 December 2014; Accepted 10 December 2014

Academic Editor: Marc Thomas

Copyright (C) 2015 Lingli Cui et al. This is an open access article distributed under the Creative Commons Attribution License, which permits unrestricted use, distribution, and reproduction in any medium, provided the original work is properly cited.

\begin{abstract}
In order to solve the problem of underdetermined blind source separation (BSS) in the diagnosis of compound fault of roller bearings, an underdetermined BSS algorithm based on null-space pursuit (NSP) was proposed. In this algorithm, the signal model of faulty roller bearing is firstly used to construct an appropriate differential operator in null space. With the constructed differential operator, the mixed signals collected by the vibration sensor are decomposed into a series of stacks of narrow band signal containing the characteristics of faulty bearing. Finally, the underdetermined problem is transformed to an overdetermined problem by combining the narrow band signals and the original mixed signals into a new group of observed signals. In this way, the separation of the mixed signals can be realized. Experiments and engineering data analyses show that the problem of underdetermined BSS can be solved effectively by this approach, and then the compound fault of the roller bearing can be separated.
\end{abstract}

\section{Introduction}

Rolling bearing is the most commonly used mechanical component in mechanical equipment and its operational status directly influences the performance of the entire machinery. In engineering practice, bearing faults may cause severe mechanical failures and huge economic losses; therefore, the fault monitoring and diagnosis of rolling bearing are of high significance. However, as a typical nonstationary signal, the vibration signal of rolling bearing contains a large amount of noises in engineering practice. The vibration signal analysis and treatment are a complex nonlinear problem. So far, many researches have been carried out on fault diagnosis of rolling bearing at home and abroad.

Fourier transform offers a bridge of the signals between the time domain and frequency domain. It is the dominant method of signal treatment. However, Fourier transform only provides the statistical average of the signals on the whole but not the local information; thus it cannot characterize the stochastic, nonstationary signals. Based on
Fourier transform, the researchers suggest a great many of time-frequency analysis methods after strenuous efforts which include wavelet analysis $[1,2]$, wavelet packet analysis [3], Kurtogram and its improved Kurtogram [4-6], Sparsogram [7], Protrugram [8], and EMD and EEMD [9-11]. Using these methods, we can gain an insight into the general and local properties of time-frequency or time-scale. It is more accurate and reliable to analyze the signals both generally and locally than to perform only time-domain or frequencydomain analysis. Most methods decompose the signals by using fixed basis function or kernel function, which directly gives rise to the uncertainty principle. When the input signals contain two modulated signals with similar frequency, a strong perturbation term will appear in the time-frequency domain. EMD method is featured by self-adaptability; however, when it is used to analyze the compound fault containing fault components of similar frequency, mode mixing and false components may occur because of the mutual disturbance between the fault features. 


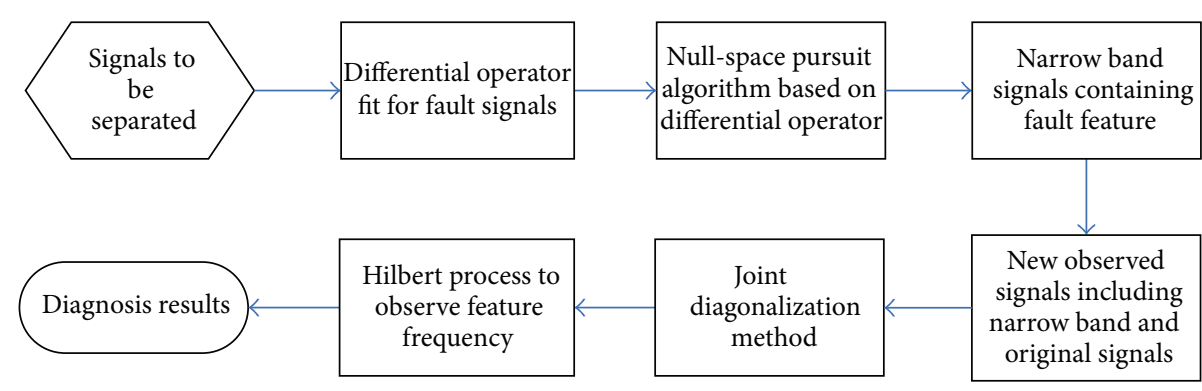

FIGURE 1: Diagram of the underdetermined BSS algorithm based on NSP.

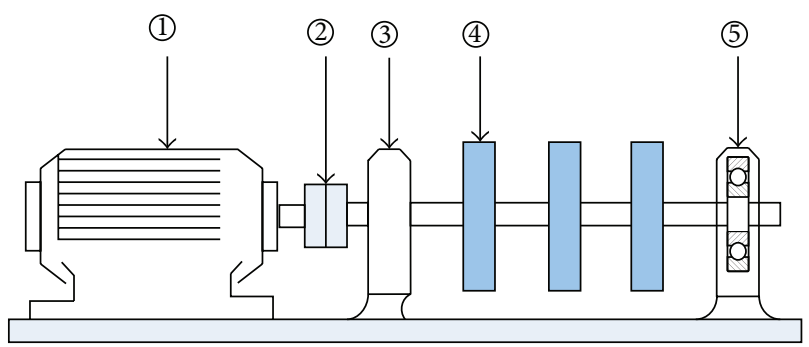

FIGURE 2: Test rig of fault bearing.

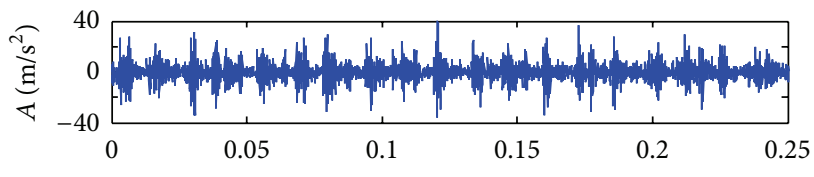

$t(\mathrm{~s})$
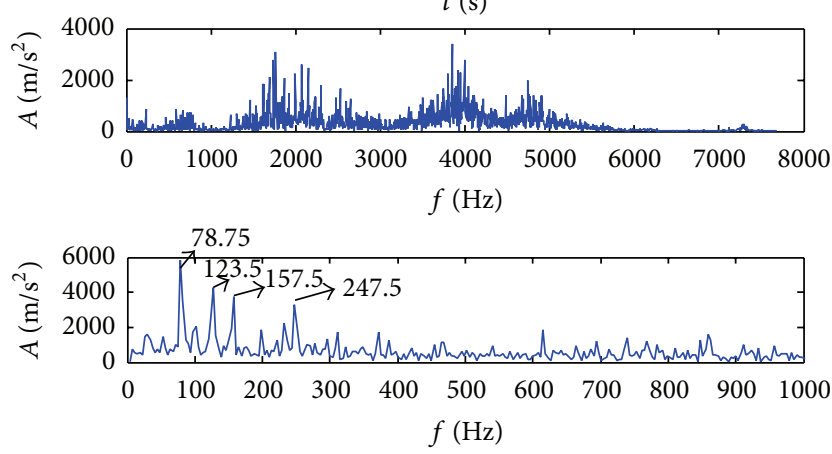

FIGURE 3: Signal waveform and frequency spectrum.

On the basis of EMD algorithm, Peng and Hwang put forward a self-adaptive decomposition algorithm utilizing the local narrow band signals and operator theory that is the null-space pursuit algorithm (NSP) [12]. This algorithm is executed by solving a regularization method for an optimization problem. The core idea of NSP algorithm is to make the local narrow band signal disappear under the action of singular local linear operator. The latter can act on the signals to extract the local narrow band components of the signals. Then the local narrow band signals obtained serve as the basic signals, which are superimposed to approximate the original signals. In this way, the self-adaptive decomposition of the signals is achieved.
Peng and Hwang carried out an optimization of this algorithm so that the frequency-modulated local narrow band signals can effectively disappear in [13]. In 2011, Hu et al. improved the NSP algorithm on this basis. Therefore, the algorithm can well extract the amplitude- and frequencymodulated local narrow band signals [14]. Xiao et al. proposed the null-space pursuit algorithm using self-adaptive third-order differential operator. The introduction of thirdorder linear differential operator makes it possible to extract the complex exponential signals, based on which the fourthorder operator is further developed [15]. The null-space pursuit algorithm is mainly applied to the field of image processing, and currently its extension of the application to other fields of engineering is under discussion. NSP is a signal separation method based on self-adaptive operator, which is derived from the vibration model of the signals to be analyzed, so the NSP has a strong self-adaptability. Until now, the algorithm is rarely used in the fault diagnosis of bearing other than in image processing [16].

In recent years some researchers have introduced BSS into compound fault diagnosis of rolling bearings and gained some progress $[17,18]$. In this paper, a new method of underdetermined BSS based on NSP is proposed to diagnose the rolling bearings compound faults.

The paper is organized as follows. Section 2 presents the mathematic model of faulty rolling bearing vibration signal. Section 3 addresses the new underdetermined BSS method based on the vibration model of faulty rolling bearing and the null-space pursuit algorithm. Section 4 presents experiment signal analysis results. The new method is validated further through the analysis of engineering example in Section 5. Finally, Section 6 concludes the paper with some remarks for the future work.

\section{Signal Vibration Model for Fault Analysis of the Rolling Bearing}

As known from literature $[19,20]$, the vibration mode of the rolling bearing can be approximately considered as a massspring-damping system. By analyzing the fault features of the bearing, the signal of the faulty bearing can be expressed as

$$
x(t)=\sum_{-\infty}^{+\infty} k e^{-n t} \cos (\omega t) \cdot \delta\left(t-a T_{0}\right),
$$


where $k$ denotes the spring coefficient; $a \in(-\infty,+\infty) ; \delta(t)$ is unit pulse function.

The vibration signals acquired by the acceleration sensor are expressed as

$$
s(t)=x(t)+n(t)
$$

where $x(t)$ is the valid signal among all signals collected; $n(t)$ is the environment noise.

The basic valid component of the faulty bearing signals is the attenuated signal of impact as follows:

$$
y(t)=k e^{-n t} \cos (\omega t)
$$

The vibration model can be approximated to a massspring-damping system. It is known that, for a mass-springdamping system, its dynamic model is expressed as follows:

$$
m y^{\prime \prime}+c y^{\prime}+k y=0,
$$

where $m$ is the mass; $y^{\prime}$ is first derivative; $y^{\prime \prime}$ is second derivative; $c$ is the damping coefficients. Let $\omega_{n}^{2}=k / m, n=$ $c / 2 m$, where $\omega_{n}$ is system frequency; then formula (4) can be written in the following form:

$$
y^{\prime \prime}+2 n y^{\prime}+\omega_{n}^{2} y=0 \text {. }
$$

The damping ratio $\xi=n / \omega_{n}$ is defined. Thus, formula (5) is rewritten:

$$
y^{\prime \prime}+2 \xi \omega_{n} y^{\prime}+\omega_{n}^{2} y=0
$$

In underdamped situation, since $\xi \ll 1$, there is $\omega=$ $\sqrt{1-\xi^{2}} \omega_{n} \approx \omega_{n}$, where $\omega$ is the angular frequency of damped vibration. Then formula (6) can be further rewritten into

$$
y^{\prime \prime}+2 \xi \omega y^{\prime}+\omega^{2} y=0
$$

Its solution is approximately expressed as

$$
y(t)=k e^{-\xi \omega t} \cos (\omega t)
$$

Given the definition of damping ratio as $\xi=n / \omega_{n}$, formula (8) is equivalent to formula (3).

Through the above analysis, it can be obtained that the basic component $y(t)=k e^{-\xi \omega t} \cos (\omega t)$ of fault signal of the rolling bearing is the solution to the second-order differential equation $y^{\prime \prime}+2 \xi \omega y^{\prime}+\omega^{2} y=0$. As known from literature [19], although the types of fault caused by the damage on different positions of rolling bearing vary, the types of fault occurring in the inner ring, outer ring, and rolling body can be all approximately considered as exponential decay function. That is to say, the basic components of these fault types are all written in the form as formula (8). The types of fault on different positions of rolling bearing conform to second-order differential equation $y^{\prime \prime}+2 \xi \omega y^{\prime}+\omega^{2} y=0$. From this, the differential operator of different fault type can be constructed specifically for the rolling bearing.
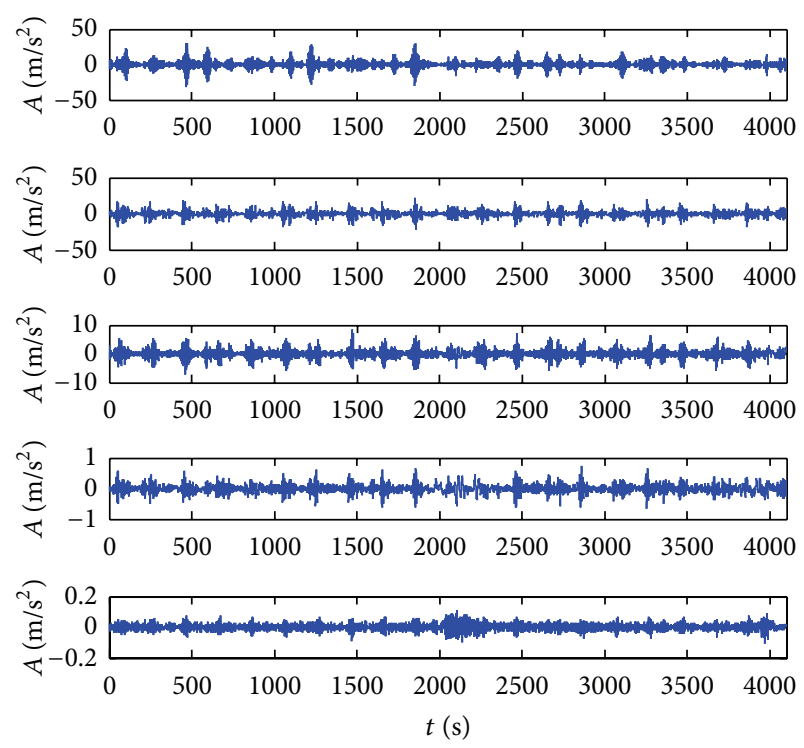

(a) Narrow band signals after NSP processing
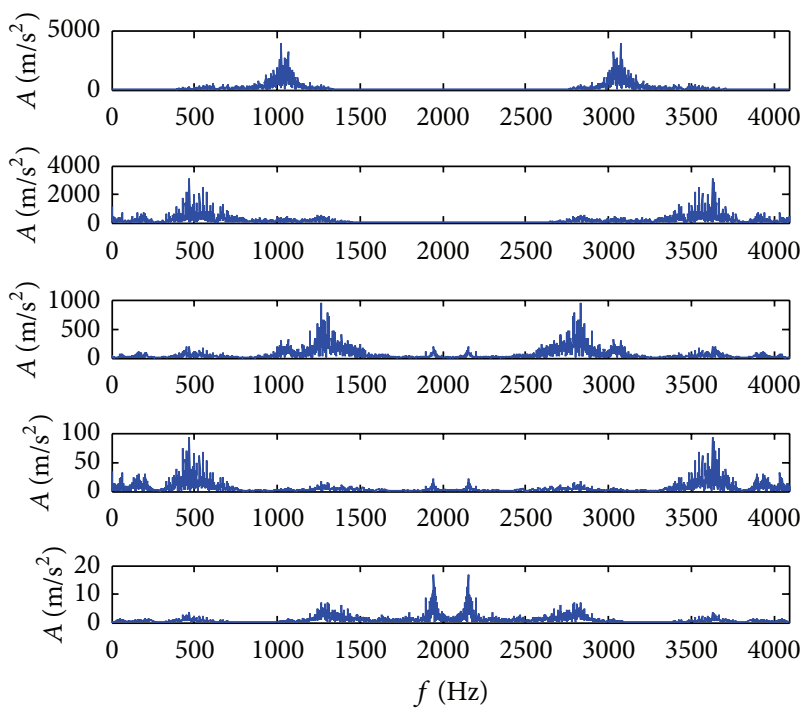

(b) The frequency spectra of narrow band signals after NSP processing

FIGURE 4: Narrow band signals after NSP processing.

\section{New Underdetermined BSS Method Based on Null-Space Pursuit}

3.1. Null-Space Pursuit Algorithm (NSP). NSP algorithm is a self-adaptive signal decomposition method based on the operator. Due to the vibration model of valid signal components among all signals to be decomposed, an appropriate operator can be estimated. By locating the valid signals among the signals to be decomposed in the null space, the operator is used to extract the narrow band signals from the signals to be decomposed that are related to the vibration model of valid signals. Usually, the signal in the form of $A(t) \cos (\omega t+\phi(t))$ is called the narrow band signals. $A(t)$ is a signal with limited bandwidth and frequency far smaller than $\omega . \phi(t)$ is a phase function. Suppose $s(t)$ is a narrow band signal. If there is an operator $T$ that makes the signal $s(t)$ equal 

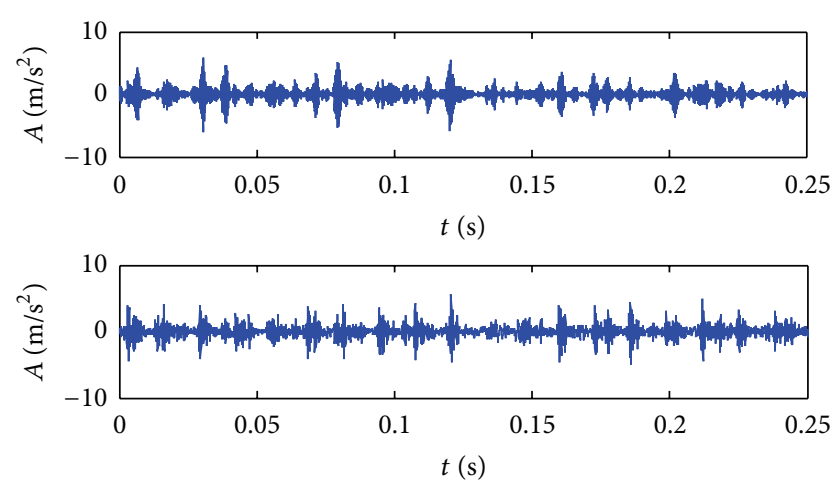

FIGURE 5: Signals after BSS and NSP processing.

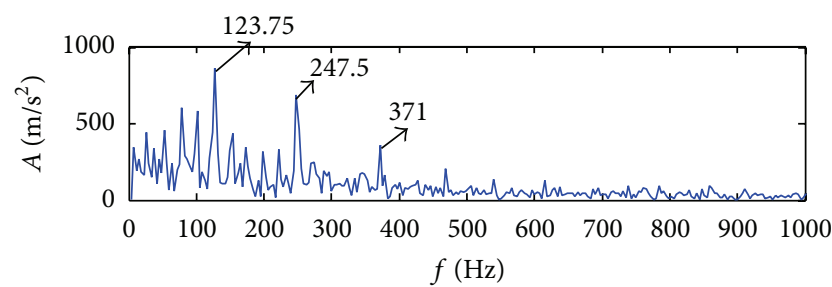

(a)

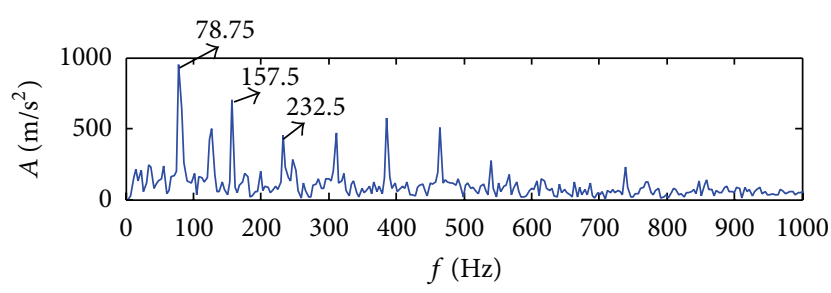

(b)

FIGURE 6: Demodulation spectra after BSS and NSP processing.

to zero, that is, $T(s(t))=0$, then it is said that the signal $s(t)$ is located in the null space of operator $T$. If the signal $S(t)$ being decomposed is treated by the operator $T$ for many times, then the components of signal $S(t)$ that is located in the null space of operator $T$ will be extracted one by one. Thus, the signal can be finally written in the following form:

$$
S(t)=\sum_{i=1}^{k} U_{i}+R_{k}
$$

where $U_{i}$ is the narrow band signal located in the null space of operator $T_{i-1}$. Here $T_{i-1}$ is the operator estimated from signal $S(t)-\sum_{k=1}^{i-1} U_{k}$. $R_{k}$ is the residual signal obtained by treating the signal $S(t)$ for $k$ times.

It is seen from the above analysis that the construction of operator in NSP algorithm is the core. For the rolling bearing, the valid components of the fault signals of the rolling bearing are located in the null space of the following operator according to formula (7):

$$
T_{f}=\frac{d^{2}}{d t^{2}}+2 \xi \omega \frac{d}{d t}+\omega^{2} .
$$

Consider a second-order operator in the following form:

$$
T=\frac{d^{2}}{d t^{2}}-\frac{2 a(t)^{\prime} d}{a(t) d t}+2\left(\frac{a(t)^{\prime}}{a(t)}\right)^{2}+\omega(t)^{2},
$$

where $a(t)$ is the envelope of signal $S(t)=a(t) \cos (\omega t)$ and $\omega$ is instantaneous frequency.

In the fault signal $y(t)=k e^{-\xi \omega t} \cos (\omega t)$ of rolling bearing, there is $a(t)=k e^{-\xi \omega t}$, which is substituted into formula (11):

$$
T_{f}=\frac{d^{2}}{d t^{2}}+2 \xi \omega \frac{d}{d t}+\omega^{2}\left(1+\xi^{2}\right) .
$$

Because $\xi \ll 1$, the value of $\xi^{2}$ is small. At this time, operator (10) is approximately equal to operator (12); thus, from (10), (11), and (12), it can be known that operator (11) is able to effectively extract the fault signals of the bearing.

The procedures of NSP algorithm proposed for the operator are as follows.

(1) The original signal $S$, the terminating thresholds $\varepsilon, \lambda_{2}^{0}$, and $\gamma^{0}$, and the initial value of $\lambda_{1}^{0}$ are inputs.

(2) Let $j=0, U_{j}=0, \lambda_{1}^{j}=\lambda_{1}^{0}$, and $\gamma^{j}=\gamma^{0}$.

(3) $\widehat{\Phi}$ is calculated by the following formula: $\widehat{\Phi}=$ $-\left(A^{T} A+\lambda_{2} M_{2}^{T} M_{2}\right)^{-1} A^{T} D_{2}(S-U)$.

(4) The parameter $\hat{\lambda}^{j+1}$ is calculated by the following formula:

$$
\hat{\lambda}^{j+1}=\frac{1}{1+\gamma^{j}} \frac{S^{T} M\left(\lambda_{1}^{j}, \gamma^{j}, T\right)^{T} S}{S^{T} M\left(\lambda_{1}^{j}, \gamma^{j}, T\right)^{T} M\left(\lambda_{1}^{j}, \gamma^{j}, T\right) S} .
$$

(5) $\widehat{U}_{j+1}$ is calculated by the following formula: $\widehat{U}_{j+1}=$ $\left(T^{T} T+\left(1+\gamma^{j}\right) \lambda_{1}^{j+1} E\right)^{-1}\left(T^{T} T S+\gamma^{j} \lambda_{1}^{j+1} S\right)$.

(6) The leakage parameter $\gamma^{j+1}$ is calculated by the following formula:

$$
\gamma^{j+1}=\frac{\left(S-\widehat{U}_{j+1}\right) S}{\left\|S-\widehat{U}_{j+1}\right\|^{2}}-1 .
$$

(7) It is determined whether $\left\|\widehat{U}_{j+1}-\widehat{U}_{j}\right\|<\varepsilon\|S\|$ is satisfied. If it is satisfied, then $\widehat{U}=\widehat{U}_{j+1}, \widehat{\lambda}_{1}=\widehat{\lambda}_{1}^{j+1}, \widehat{\gamma}=$ $\gamma^{j+1}$, and $\widehat{\alpha}=\widehat{\alpha}_{j}$.

$R=(S-\widehat{U})(1+\widehat{\gamma})$ and $U=S-R$ are output. Otherwise, let $j=j+1$; then return to step (3), where $A=\left[A_{D_{1}(S-U)}, A_{S-U}\right], M_{1}=\left[D_{1}^{T}, E^{T}\right]^{T}, M_{2}=\left[{ }^{D_{2}}\right]$, and $B_{\Phi}=\left\lfloor A_{p}, A_{q}\right\rfloor$.

$p$ and $q$ can be obtained from $\widehat{\Phi}=\left[p^{T}, q^{T}\right]^{T}, T=D_{2}+$ $B_{\widehat{\Phi}} M_{1}$, and $M\left(\lambda_{1}^{j}, \gamma^{j}, T\right)=\left(T^{T} T+(1+\widehat{\gamma}) \lambda_{1} E\right)^{-1}$.

Matrix $A_{x}$ is the diagonal matrix with the diagonal element being $X$ vector. $E$ is identity matrix. $D_{1}$ and $D_{2}$ are first-order and second-order differential matrices; $\hat{\lambda}_{1}$ is Lagrange parameter; $\hat{\gamma}$ is the parameter used to determine the information amount of $S-R$ in the null space of $T_{c}$. The initial value of parameter in the algorithm is configured based on experience. 


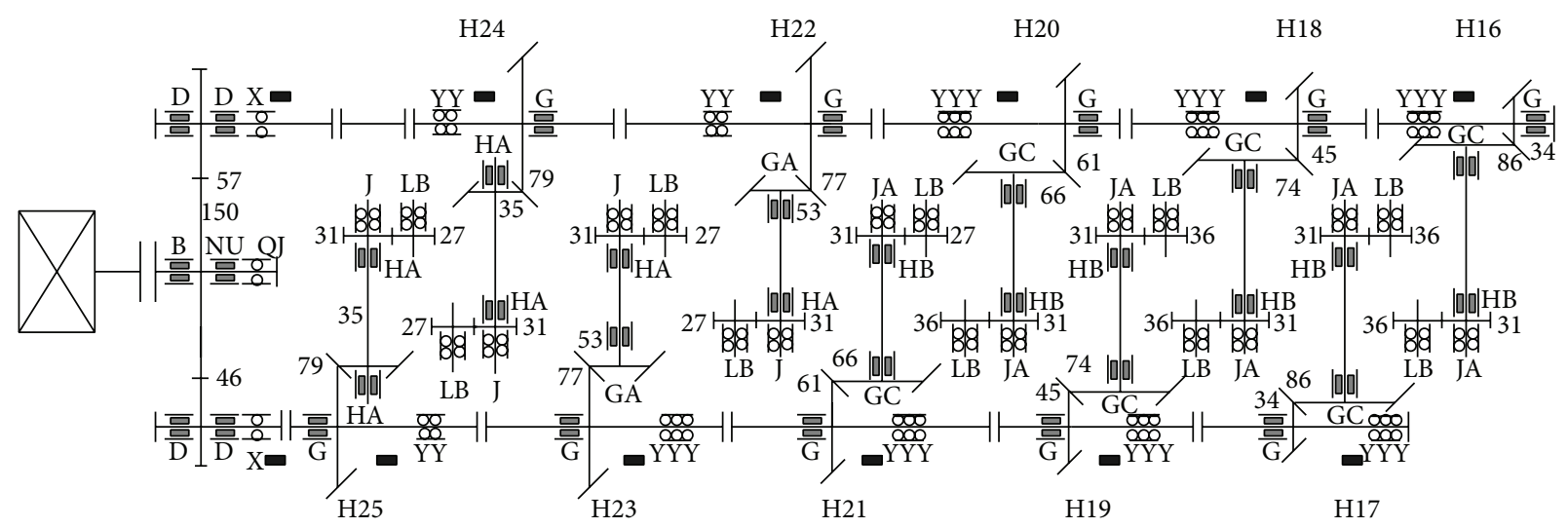

- Measurement points

Figure 7: Driving chain of gearbox.

3.2. BSS Algorithm. BSS algorithm is a method of signal processing used to estimate the unknown source signal and parameter of unknown hybrid channel through only the observed signals collected by the sensor. Currently, the most widely used methods of BSS include independent component analysis (ICA) and the approximate joint diagonalization algorithm based on eigenmatrix proposed by Cui et al. and Cardoso [21, 22], which is employed in this study.

The procedure is shown as follows.

(1) Whitening process is performed on the observed signal $x(t)$. Suppose $x(t)=\left(x_{1}(t), x_{2}(t), \ldots, x_{m}(t)\right)^{T}$ contains $m$ observed signals, $s(t)=\left(s_{1}(t), s_{2}(t), \ldots, s_{n}(t)\right)^{T}$ represents the $n$ unknown source signals, and $A$ is a full-rank mixing matrix $(m \geq n)$ with $m \times n$. Then the model of BSS is shown as follows:

$$
x(t)=A s(t)
$$

The autocorrelation matrix of the observed signal $x(t)$ is defined as follows:

$$
R_{x}=E\left[x(t) x(t)^{*}\right]
$$

where the superscript $*$ denotes the complex conjugate and the eigenvalue of $R_{x}$ is decomposed into the characteristic roots and the corresponding eigenvectors. Here $\lambda_{1}, \lambda_{2}, \ldots, \lambda_{N}$ denote the top $N$ large characteristic roots and $q_{1}, q_{2}, \ldots, q_{N}$ denote the corresponding eigenvectors. Suppose the interference is white noise; then the estimation of noise variance $\delta^{2}$ is the average of the $m-n$ smallest eigenvalues of the autocorrelation matrix $R_{x}$. The whitening signal $z(t)=\left(z_{1}(t), z_{2}(t), \ldots, z_{n}(t)\right)^{T}$ can be obtained with the following formula:

$$
z_{i}(t)=\left(\lambda_{i}-\delta^{2}\right)^{-1 / 2} q_{i}^{*} x(t) \quad 1 \leq i \leq n
$$

Then the whitening matrix is obtained as follows:

$$
W=\left(\left(\lambda_{1}-\delta^{2}\right)^{-1 / 2} h_{1}, \ldots,\left(\lambda_{n}-\delta^{2}\right)^{-1 / 2} h_{n}\right)^{H}
$$

Here the superscript $H$ denotes the transposition of complex conjugate. The whitening observed signal can be obtained by multiplying formula (15) by the whitening matrix $W$ from the left:

$$
z(t)=W x(t)=W A s(t)=U s(t) .
$$

The signal after whitening is the mixture of unitary matrix of the source signal. In this way, the problem of determining the mixing matrix $A$ is transformed to find the unitary matrix $U$.

(2) The joint diagonalization process is performed on the whitened signal to determine the unitary matrix $U$, so as to obtain the estimation of source signal. In this study, the approximate joint diagonalization algorithm based on eigenmatrix by Cardoso is used. For any matrix $M$ with $n \times n$, the fourth-order cumulant matrix $Q_{z}(M)$ of $z(t)$ is defined as follows:

$$
\begin{array}{r}
{\left[Q_{z}(M)\right]_{i j} \Longleftrightarrow \sum_{k=1}^{n} \sum_{l=1}^{n} \operatorname{cum}\left(z_{i}, z_{j}^{*}, z_{k}, z_{l}^{*}\right) m_{k l}} \\
i, j=1,2, \ldots, n,
\end{array}
$$

where $\operatorname{cum}\left(z_{i}, z_{j}^{*}, z_{k}, z_{l}^{*}\right)$ is the fourth-order cumulant matrix of four components $i, j, k, l$ in the whitening matrix $z(t) . m_{k l}$ is the element on the $k$ th row and the $l$ th column of the matrix $M$.

Moreover, with the optimization, the matrix $U$ is obtained by performing the joint diagonalization on the fourth-order cumulant of the whitening matrix $z(t)$.

In the calculating process, the quadratic sum of the diagonal components in matrix $U^{H} Q_{z}\left(M_{r}\right) U$ is used as a measure shown as

$$
f(U)=\sum_{r=1}^{n}\left|\operatorname{diag}\left(U^{H} Q_{z}\left(M_{r}\right) U\right)^{2}\right| .
$$

The unitary matrix $U$ can be obtained by maximizing the formula above. Then the estimation of source signal can be achieved:

$$
y=U^{H} W x(t)
$$




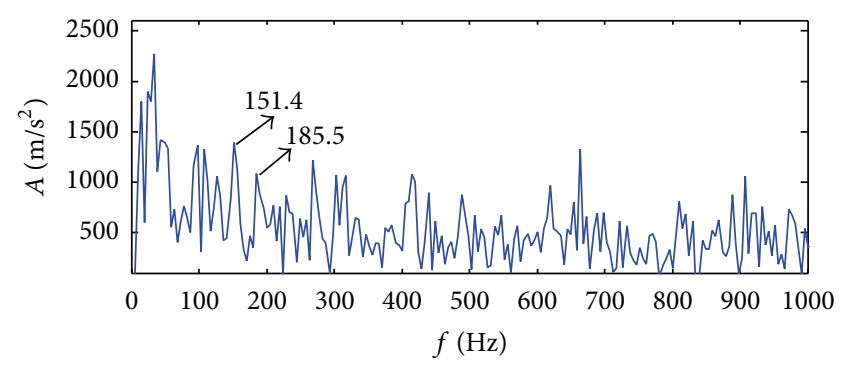

FIGURE 8: Hilbert demodulation spectrum.

3.3. Underdetermined BSS Algorithm Based on Null-Space Pursuit. According to the analysis of BSS and NSP, the mixed signals can be separated when the number of the original signals is larger than the observed signals, and they can be decomposed into a series of stacks of narrow band signal which contains the bearing fault information only if the operator of NSP is selected appropriately. Therefore, the mixed signals can be preprocessed by NSP algorithm and obtain several observed signals which are combined with the original observed signals to get a new group of observed signals. Thus, the number of the observed signals is larger than the original signals. Furthermore, the underdetermined problem is transformed into overdetermined problem to realize the separation of the bearing fault characteristics. The method is performed as follows.

(1) The differential operator is constructed according to the vibration model of signal of faulty roller bearing.

(2) With the constructed operator, the observed signals are decomposed into a series of stacks of narrow band signal which contains characteristics of the faulty bearing.

(3) The above narrow band signals and the original observed signals are combined into a new group of observed signals.

(4) The whitening matrix and the observed matrix after whitening are obtained by prewhitening the new observed signals.

(5) The joint diagonalization based on eigenmatrix is performed on the signals obtained in step (4) to determine the unitary matrix $U$, so as to obtain the estimation of source signal.

(6) The source signal can be estimated on which the Hilbert process is conducted to obtain the characteristics of faulty bearings.

The algorithm flow is shown as Figure 1.

\section{Experimental Research}

The experimental system consists of bearing test stand, HG3528A data acquisition instrument, and laptop. The test stand (shown as Figure 2) is connected to the shaft with the rotor (4) via the flexible coupling (2) using the threephase asynchronous motor (1). The shaft is supported by two
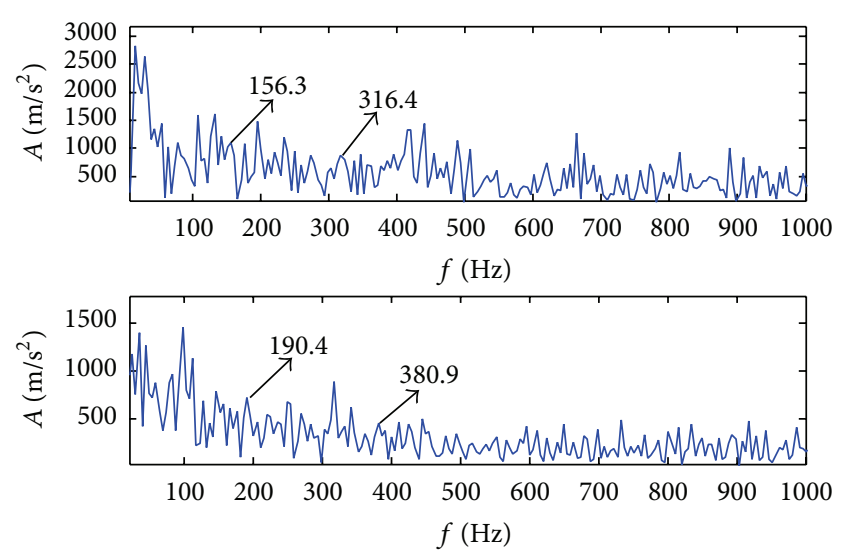

FIGURE 9: Spectrum with BSS based on NSP processing.

6307 bearings: (3) is a normal bearing and (5) is the bearing with different pitting modes. The rotating speed of motor is $R=1496 \mathrm{r} / \mathrm{min}$, the outer diameter of the bearing is $D=$ $80 \mathrm{~mm}$, and the inner diameter is $d=35 \mathrm{~mm}$. The number of rolling elements is $Z=8$, and the contact angle is $\alpha=0$. According to the above parameters and literature [20, 21], it can be calculated that the feature frequency of the fault on the outer ring of the bearing is $78.7282 \mathrm{~Hz}$, that on the inner ring is $123.738 \mathrm{~Hz}$, and that on the rolling element is $99.38 \mathrm{~Hz}$. The sampling frequency is $15360 \mathrm{~Hz}$, and the length of the analyzed signal is 4096 points.

When there are faults on both the inner and outer ring of the bearing, the observed signal is shown as Figure 3. It can be seen from the frequency spectrum of Figure 3 that the faults simultaneously occur on the inner ring and the outer ring of the bearing. There are two source signals in the experiment, the fault signals of inner ring and outer ring, while there is only one observed signal. This is a problem of the undetermined BSS, which cannot be solved by the conventional BSS algorithm. The proposed method is adopted to separate the signals. First, NSP is used to extract the observed signal for five times, and five narrow band signals are obtained. The narrow band signals obtained above contain the bearing fault characteristics information because the differential operator is constructed according to the bearing fault modeling. The five narrow band signals and their frequency spectrums are shown in Figure 4 . The five narrow band signals containing the bearing fault characteristics and the original observed signal are combined into a new group of observed signals, so that the undetermined BSS issue is transformed into an overdetermined BSS. The separation result is shown in Figure 5. The separated signal is subject to Hilbert demodulation analysis, and the obtained demodulation spectrum is shown as Figure 6. As shown in Figure 6(a), there is a fault on the inner ring of the bearing, and the peak value occurs at $123.75 \mathrm{~Hz}$, which is very close to the frequency of the fault on the inner ring of the bearing, $122.738 \mathrm{~Hz}$. In addition, there are also peak values at other multiple frequencies, $247.5 \mathrm{~Hz}$ and $371.3 \mathrm{~Hz}$, which shows the correctness of diagnosis. It can be seen from Figure 6(b) that there is a fault on the outer ring of the bearing. The peak value occurs at $78.75 \mathrm{~Hz}$, which is very close to the frequency 


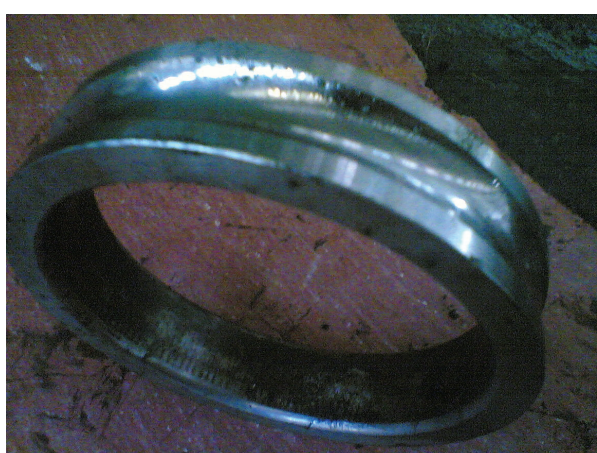

(a) Inner-race pitting

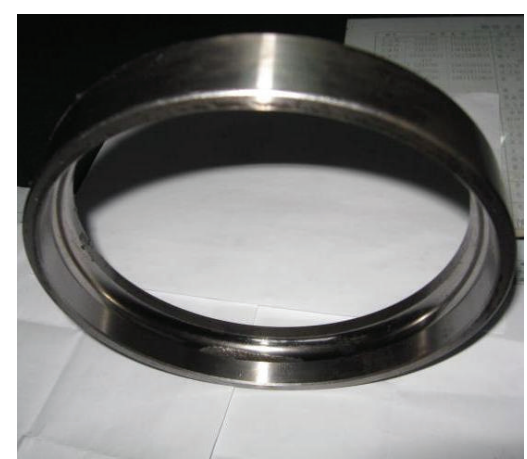

(b) Outer-race pitting

FIgURE 10: Pitting faults at the bearing of shaft I at the north of the speed-up gearbox.

of the fault on the outer ring of the bearing, $76.728 \mathrm{~Hz}$, and there are peak values at the multiple frequencies, $157.5 \mathrm{~Hz}$ and $232.5 \mathrm{~Hz}$. Therefore, the fault on the outer ring of the bearing is diagnosed. Thus, the method successfully separates the composite fault of inner and outer ring of the roller bearing, so as to implement the diagnosis of composite fault on the roller bearing.

\section{Engineering Application}

In order to verify the effectiveness of the proposed method, it is applied to engineering practice.

Figure 7 shows the drive system of high-speed wire gearbox in a steel plant. The on-site test from January 27, 2009, shows that the peak value of the horizontal measuring point at the north output end of the gearbox of the vibration data began to rise from January 21. This means that the operation situation was deteriorating. It was preliminarily diagnosed that there is a fault at the north measuring point of the gearbox.

The given condition is that the fault feature frequencies of outer ring, inner ring, and rolling element of the bearing measured (indicated by the arrow in Figure 7) were $156.75 \mathrm{~Hz}$, $191.5833 \mathrm{~Hz}$, and $138.5546 \mathrm{~Hz}$, respectively. Besides, the rotating speed of the bearing was $951 \mathrm{r} / \mathrm{min}$.

First, the Hilbert demodulation is conducted to analyze the signal, and the obtained frequency spectrum is shown as Figure 8. According to the chart, only the frequencies $151 \mathrm{~Hz}$ and $185 \mathrm{~Hz}$ are most close to the fault feature frequency. However, the fault type of the measured object cannot be determined. The outcome from the processing with the proposed algorithm is shown as Figure 9. The frequency that is extremely close to the fault feature frequency of inner ring and outer ring and its double frequency can be seen. This means that there are faults at both the inner ring and the outer ring of the analyzed bearing. After taking out the fault bearing, it was found that there was pitting on the inner and outer ring, as shown in Figure 9. This further illustrates the effectiveness of the proposed method.

Pitting faults at the bearing of shaft I at the north of the speed-up gearbox were found as shown in Figure 10.

\section{Conclusions}

The mixed signals can be separated effectively by the traditional BSS only if the number of the observed signals is larger than the original signals. To solve the underdetermined problem in single channel bearing fault diagnosis, a new BSS algorithm based on null-space pursuit algorithm was proposed. The null-space pursuit algorithm is an adaptive signal decomposition algorithm which can separate mixed signals into several narrow band signals effectively with effective operator constructed. In this paper, firstly, the signal model of faulty bearing is analyzed and the appropriate operator is constructed according to the characteristics of faulty bearings. Secondly, the mixed signals are preprocessed by the operator constructed above to get a group of narrow band signals that contains fault features. Thirdly, a new group of observed signals are obtained by combining the narrow band signals with the original observed signals; thus the separation of the bearing fault characteristics is realized. Finally, the engineering signals and the experiment signals are used to validate the proposed method. The results show that the traditional underdetermined problem of BSS is solved and the bearing fault characteristics are separated successfully. Therefore, diagnosis of roller bearings compound fault using underdetermined blind source separation algorithm based on null-space pursuit is effective.

\section{Conflict of Interests}

The authors declare that there is no conflict of interests regarding the publication of this paper.

\section{Acknowledgment}

This work is supported by the National Natural Science Foundation of China (51175007 and 51075023).

\section{References}

[1] J. Rafiee, M. A. Rafiee, and P. W. Tse, "Application of mother wavelet functions for automatic gear and bearing fault diagnosis," Expert Systems with Applications, vol. 37, no. 6, pp. $4568-$ 4579, 2010. 
[2] S. Abbasion, A. Rafsanjani, A. Farshidianfar, and N. Irani, "Rolling element bearings multi-fault classification based on the wavelet denoising and support vector machine," Mechanical Systems and Signal Processing, vol. 21, no. 7, pp. 2933-2945, 2007.

[3] N. G. Nikolaou and I. A. Antoniadis, "Rolling element bearing fault diagnosis using wavelet packets," NDT and E International, vol. 35, no. 3, pp. 197-205, 2002.

[4] J. Antoni, "Fast computation of the kurtogram for the detection of transient faults," Mechanical Systems and Signal Processing, vol. 21, no. 1, pp. 108-124, 2007.

[5] Y. G. Lei, J. Lin, Z. J. He, and Y. Y. Zi, "Application of an improved kurtogram method for fault diagnosis of rolling element bearings," Mechanical Systems and Signal Processing, vol. 25, no. 5, pp. 1738-1749, 2011.

[6] D. Wang, P. W. Tse, and K. L. Tsui, "An enhanced Kurtogram method for fault diagnosis of rolling element bearings," Mechanical Systems and Signal Processing, vol. 35, no. 1-2, pp. 176-199, 2013.

[7] P. W. Tse and D. Wang, "The design of a new sparsogram for fast bearing fault diagnosis: part 1 of the two related manuscripts that have a joint title as 'Two automatic vibration-based fault diagnostic methods using the novel sparsity measurementparts 1 and 2," Mechanical Systems and Signal Processing, vol. 40, no. 2, pp. 499-519, 2013.

[8] T. Barszcz and A. JabŁoński, "A novel method for the optimal band selection for vibration signal demodulation and comparison with the Kurtogram," Mechanical Systems and Signal Processing, vol. 25, no. 1, pp. 431-451, 2011.

[9] J. Zhang, R. Yan, R. X. Gao, and Z. Feng, "Performance enhancement of ensemble empirical mode decomposition," Mechanical Systems and Signal Processing, vol. 24, no. 7, pp. 2104-2123, 2010.

[10] D. Wang, W. Guo, and P. W. Tse, "An enhanced empirical mode decomposition method for blind component separation of a single-channel vibration signal mixture," Journal of Vibration and Control, 2014.

[11] W. Guo and P. W. Tse, "A novel signal compression method based on optimal ensemble empirical mode decomposition for bearing vibration signals," Journal of Sound and Vibration, vol. 332, no. 2, pp. 423-441, 2013.

[12] S. Peng and W.-L. Hwang, "Adaptive signal decomposition based on local narrow band signals," IEEE Transactions on Signal Processing, vol. 56, no. 7, pp. 2669-2676, 2008.

[13] S. L. Peng and W. L. Hwang, "Null space pursuit: an operatorbased approach to adaptive signal separation," IEEE Transactions on Signal Processing, vol. 58, no. 5, pp. 2475-2483, 2010.

[14] X. Y. Hu, S. L. Peng, and W.-L. Hwang, "Operator based multicomponent AM-FM signal separation approach," in Proceedings of the IEEE International Workshop on Machine Learning for Signal Processing (MLSP '11), pp. 1-6, IEEE, September 2011.

[15] W. W. Xiao, W. J. Luan, and S. L. Peng, "Null space pursuit based on three order linear differential operator," System Engineering-Theory \& Practice, vol. 33, no. 5, pp. 1283-1288, 2013 (Chinese).

[16] D. Zhu, Q. Gao, D. Sun, Y. Lu, and S. Peng, "A detection method for bearing faults using null space pursuit and S transform," Signal Processing, vol. 96, pp. 80-89, 2014.

[17] C. Servière and P. Fabry, "Principal component analysis and blind source separation of modulated sources for electromechanical systems diagnostic," Mechanical Systems and Signal Processing, vol. 19, no. 6, pp. 1293-1311, 2005.
[18] Z. Li, X. Yan, Z. Tian, C. Yuan, Z. Peng, and L. Li, "Blind vibration component separation and nonlinear feature extraction applied to the nonstationary vibration signals for the gearbox multi-fault diagnosis," Measurement: Journal of the International Measurement Confederation, vol. 46, no. 1, pp. 259-271, 2013.

[19] F. Cong, J. Chen, G. Dong, and M. Pecht, "Vibration model of rolling element bearings in a rotor-bearing system for fault diagnosis," Journal of Sound and Vibration, vol. 332, no. 8, pp. 2081-2097, 2013.

[20] H. B. Mei, Monitoring and Diagnosis of Rolling Bearing, China Machine Press, Beijing, China, 1996, (Chinese).

[21] L. Cui, J. Wang, and S. Lee, "Matching pursuit of an adaptive impulse dictionary for bearing fault diagnosis," Journal of Sound and Vibration, vol. 333, no. 10, pp. 2840-2862, 2014.

[22] J.-F. Cardoso, "Blind signal separation: statistical principles," Proceedings of the IEEE, vol. 86, no. 10, pp. 2009-2025, 1998. 

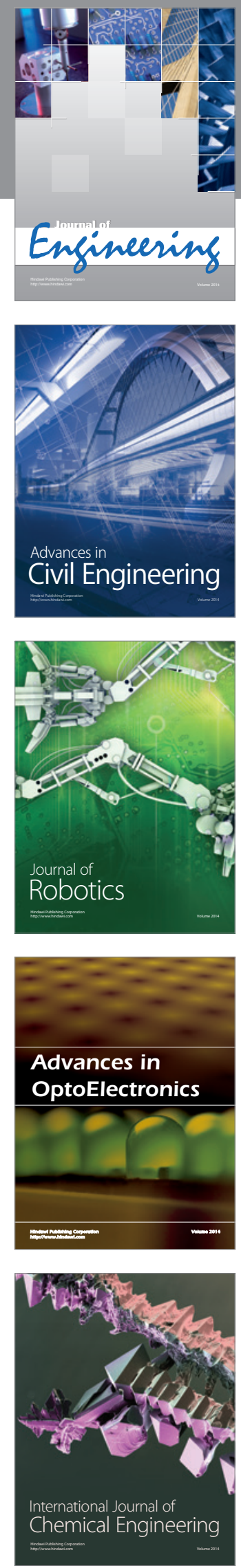

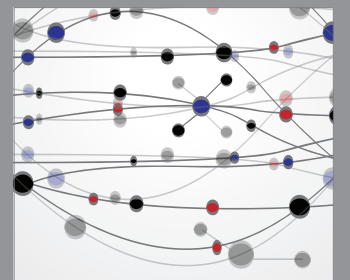

The Scientific World Journal
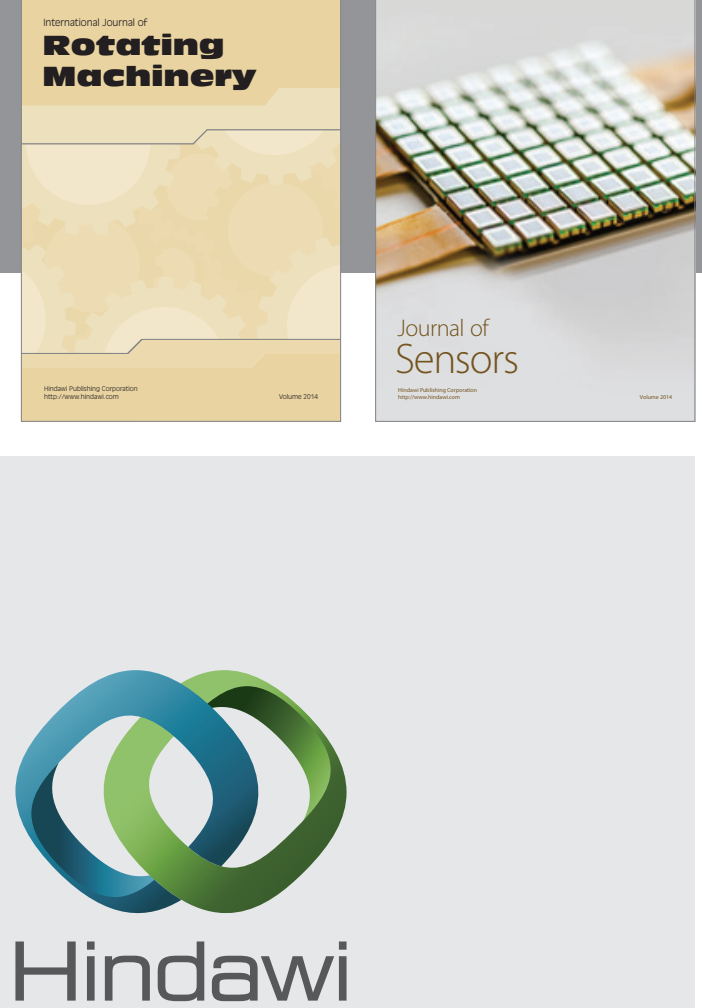

Submit your manuscripts at http://www.hindawi.com
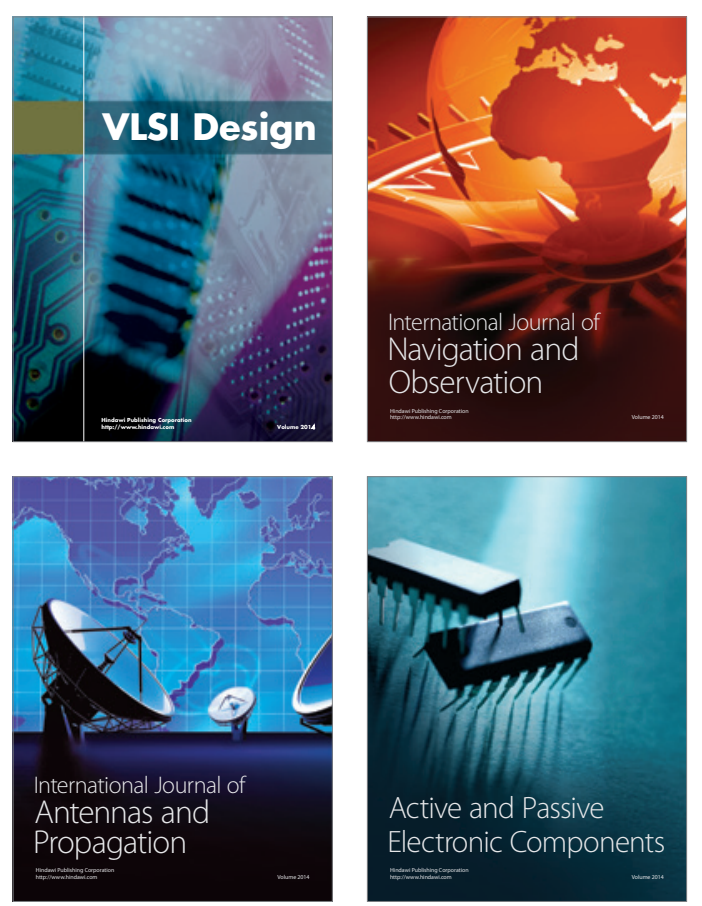
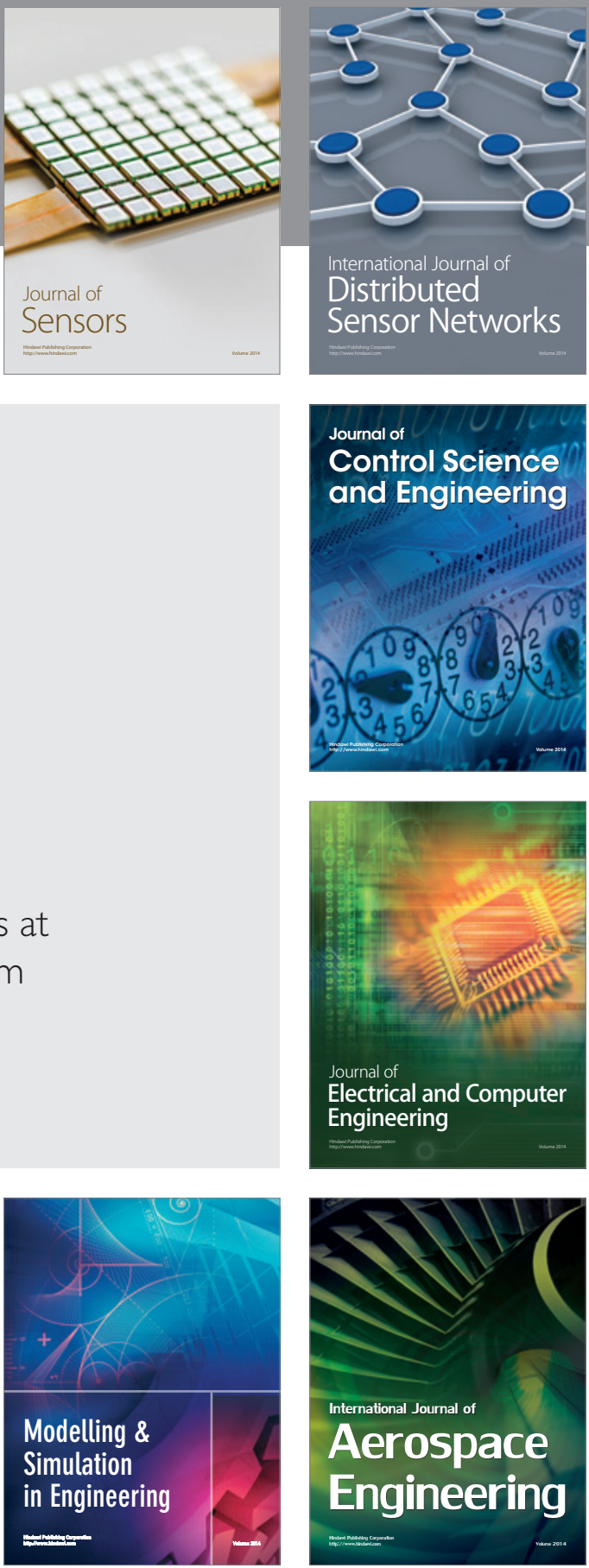

Journal of

Control Science

and Engineering
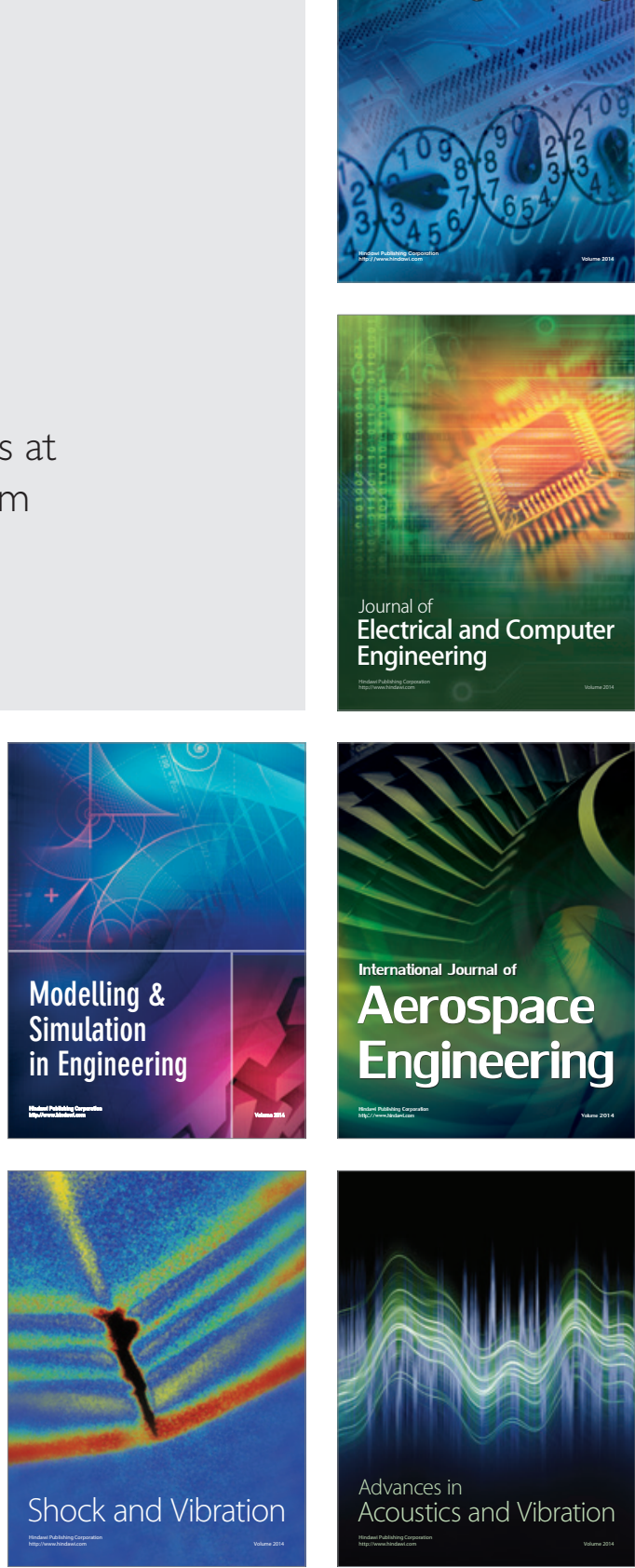